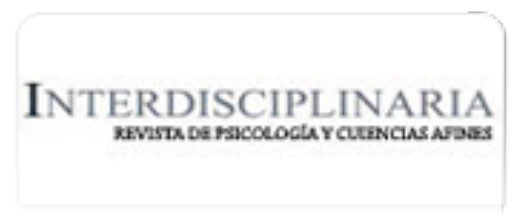

Interdisciplinaria

ISSN: 0325-8203

ISSN: 1668-7027

interdisciplinaria@fibercorp.com.ar

Centro Interamericano de Investigaciones Psicológicas y

Ciencias Afines

Argentina

Hauser, María Paulina; Milán, Teresita Ana; Oiberman, Alicia

Interacciones madre-bebé de alto riesgo. Una aplicación del análisis de clúster en dos contextos de interacción: UCIN y Consultorio de Seguimiento

Interdisciplinaria, vol. 35, núm. 2, 2018, Julio-, pp. 543-559

Centro Interamericano de Investigaciones Psicológicas y Ciencias Afines

Argentina

Disponible en: https://www.redalyc.org/articulo.oa?id=18058785018

Cómo citar el artículo

Número completo

Más información del artículo

Página de la revista en redalyc.org

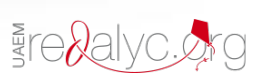

Sistema de Información Científica Redalyc Red de Revistas Científicas de América Latina y el Caribe, España y Portugal Proyecto académico sin fines de lucro, desarrollado bajo la iniciativa de acceso abierto 


\title{
Interacciones madre-bebé de alto riesgo. Una aplicación del análisis de clúster en dos contextos de interacción: UCIN y Consultorio de Seguimiento
}

\author{
Mother-high-risk infant interactions. An application of cluster \\ analysis in two contexts of interaction: NICU and Follow-up Office
}

\author{
María Paulina Hauser*, Teresita Ana Milán** y Alicia Oiberman***
}

\begin{abstract}
* Doctora en Psicología. Jefa de Trabajos Prácticos en Psicopatología I y II, Facultad de Psicología, Universidad Nacional de San Luis (UNSL), Argentina. Becaria postdoctoral del Consejo Nacional de Investigaciones Científicas y Técnicas (CONICET). E-mail: mphauser@unsl.edu.ar

**Doctora en Psicología. Psicóloga Clínica. E-mail: tmilan@unsl.edu.ar ***Doctora en Psicología. Investigadora Independiente del Consejo Nacional de Investigaciones Científicas y Técnicas (CONICET). Profesora Titular de Psicología Evolutiva I, Universidad de Palermo. Responsable del Postgrado en Psicología Perinatal, Facultad de Psicología, Universidad de Buenos Aires (UBA).

E-mail: aoiberma@psi.uba.ar
\end{abstract}

Facultad de Psicología, Universidad Nacional de San Luis (UNSL). San Luis, Argentina.

\section{Resumen}

Las interacciones tempranas pueden presentarse de manera diferente cuando se trata de bebés de alto riesgo, que requieren de cuidados especiales al nacer y deben permanecer internados durante su período neonatal. El objetivo de la investigación que se expone fue conocer el modo en que se desplegaban las interacciones madrebebé de alto riesgo durante la internación en la Unidad de Cuidados Intensivos Neonatales (UCIN) y en el Consultorio de Seguimiento para Niños de Alto Riesgo hasta los 12 meses de edad gestacional corregida de los infantes.

La muestra estuvo formada por 36 díadas mamá-bebé de alto riesgo. Se administraron la Escala de observación de vínculo madre-bebé internado en UCIN y la Escala de observación del vínculo madre-hijo. Se utilizó la técnica estadística de Análisis de Clúster, que permitió agrupar a las díadas madre-bebé en función de las características de las interacciones en los dos contextos de observación.

Los resultados permitieron distinguir díadas que tuvieron dificultades para interactuar, tanto en la UCIN como durante los controles médicos en el Consultorio de Seguimiento, otras que lograron mantener interacciones gratificantes en la UCIN y en el Consultorio de Seguimiento para Niños de Alto Riesgo, y otras que interactuaron de manera alternante durante el primer año de vida.

La utilización de la técnica estadística de Análisis de Clúster resulta novedosa en nuestro medio y en especial en el área de la Psicología Perinatal. Esta técnica permite visualizar y agrupar de manera estadística a las interacciones en función de sus características.

Palabras clave: Interacciones; Madre-bebé de alto riesgo; Unidad de Cuidados Intensivos Neonatales; Consultorio de Seguimiento para Niños de Alto Riesgo; Análisis de Clúster.

\begin{abstract}
Early interactions may be different in the case of high-risk infants who require special care at birth and must remain hospitalized during their neonatal period. These babies are at highrisk for sequelae, especially those with a history
\end{abstract}


of prematurity, low birth weight, neonatal conditions and important psychosocial problems. At the birth of a child with some difficulty, parents experience a multiplicity of feelings that result in the vicissitudes of interactions and the mother-father-child bond. In these cases, there is a greater relational risk, which is why there are greater possibilities for the appearance of dysfunctional bonding modalities. The objective of the research that is exposed was to know the way in which mother-high-risk infant interactions were deployed during hospitalization at the Neonatal Intensive Care Unit (NICU) and in the Follow-up Office for High-Risk Children up to 12 months corrected gestational age of infants.

The sample is made up of 36 mother-highrisk baby dyads. We used the Mother-Child Bond Observation Scale in the NICU during the period of hospitalization of the baby in the NICU. Observations were made when the mother entered the NICU to see her child while in the incubator. The first observation of the dyad should be made during the first month of life of the baby, performing the following with intervals of two or three days in order to know if there were variations with the course of hospitalization time, maturation and growth of the baby. At follow-up, spontaneous mother-child interactions that occurred in the natural observational context of the High-Risk Child FollowUp Office were evaluated using the MotherChild Bond Observation Scale. It was observed monthly to each dyad during the 15-25 minutes of the pediatric visit. The statistical technique of Cluster Analysis was used, which allowed to group the mother-infant dyads according to the characteristics of the interactions in the two observational contexts.

The results allowed us to distinguish dyads who had difficulty interacting both in the NICU and during the medical exam at the Follow-up Office; other dyads were able to maintain rewarding interactions in the NICU and in the Follow-up Office for High-risk Children. Finally, other dyads interacted alternately during the first year of life, either because they established satisfactory interactions during the infant's hospitalization in the NICU, although later these interactions became significantly impoverished during the first year of life. Or had difficulties in interacting in neonatal therapy, which decreased considerably after discharge, with frequent and rewarding interactions observed during the infant's follow-up in the first year of life.

The importance of the application of Cluster Analysis in the field of research in Psychology lies in the possibility of visualizing and grouping in a statistical way, in our case, the interactions according to their characteristics, in two different moments such as Hospitalization in NICU and in the Office of Follow-up for High-Risk Children. The use of the statistical technique of Cluster Analysis is novel in our environment and especially in the area of Perinatal Psychology.

Key words: Interactions; Mother-High-risk baby; Neonatal Intensive Care Unit; Follow-up Office for High-Risk Children; Cluster Analysis

\section{Introducción}

La relación del bebé con sus progenitores ha sido entendida y valorada de diferentes maneras a lo largo de la historia. El logro de una conexión emocional sólida será la base para un desarrollo adecuado en los niños, mientras que las interferencias y fallas en la vinculación pueden generar efectos negativos en la salud mental, tanto a corto como a largo plazo (Raznoszczyk de Schejtman, 2005). La relación que se establezca con los padres o cuidadores relevantes en los primeros años de vida, se expresa principalmente a través de los cuidados cotidianos y del juego como un modo privilegiado de intercambio entre el niño y los adultos (Leonardelli, Vernengo, Wainer \& Duhalde, 2009).

Las interacciones tempranas se presentan de manera diferente cuando se trata de bebés de alto riesgo, que deben permanecer internados durante su período neonatal. Se entiende por bebés de alto riesgo a aquellos que al momento de nacer, ya sea por circunstancias prenatales, como perinatales o posnatales, requieren de cuidados especiales, indispensables para lograr su supervi- 
vencia, así como para disminuir la posibilidad de aparición de trastornos funcionales graves durante la infancia (Nikodem, 2009). La Guía de Seguimiento del Recién Nacido de Riesgo (2003) agrega que estos bebés tienen un riesgo elevado de presentar secuelas, especialmente aquellos con antecedentes de prematurez, bajo peso al nacer, patologías neonatales y problemas psicosociales importantes.

Pose (2004) señala que ante el nacimiento de un hijo con alguna dificultad, los padres experimentan una multiplicidad de sentimientos que redundan en las vicisitudes de las interacciones y del vínculo madre-padre-hijo. En estos casos, existe un riesgo relacional mayor, por lo que hay mayores posibilidades de aparición de modalidades vinculares disfuncionales. El momento de encuentro (Brazelton \& Nugent citado por Bruschweiler-Stern, 2009) se verá postergado ante el nacimiento de un bebé de alto riesgo, quien deberá ser trasladado a una Unidad de Cuidados Intensivos Neonatales (UCIN) donde permanecerá internado. Davim, Enders y Silva (2010) afirman que cuando una familia enfrenta un nacimiento prematuro se produce una situación de angustia e incertidumbre por el riesgo en la salud del bebé. Las madres experimentan predominantemente sentimientos penosos, de culpa, decepción, frustración e inseguridad.

Guillaume y colaboradores (2013) sostienen que durante las primeras semanas de internación, el bebé prematuro y sus padres enfrentan dificultades para el establecimiento de las interacciones y del vínculo temprano. Flacking y colaboradores (2012) enfatizan la importancia de la cercanía física y emocional entre el recién nacido prematuro y sus padres en la UCIN, lo que ha demostrado tener efectos positivos sobre el desarrollo cerebral del niño, sobre el bienestar psicológico de los padres y sobre las interacciones padre-hijo.

Se ha puesto en evidencia que las madres de bebés prematuros presentan menos interacciones positivas con sus hijos que las madres de los bebés a término; y que las ma- dres de bebés de muy bajo peso que permanecieron separadas por más tiempo presentan dificultades en las conductas de apego en comparación con las madres de los bebés nacidos a término (Korja, Latva \& Lehtonen, 2012; Feldman, Weller, Leckman, Kuint \& Eidelman, 1999; Caskey, Stephens, Tucker \& Vohr, 2011, citados por Flacking et al., 2012). Los resultados de una investigación reciente sugieren que estadísticamente el transcurso del tiempo de internación no incidiría en la calidad de las interacciones diádicas en la UCIN. La mejora en las interacciones a medida que transcurren algunos días podría deberse a que las díadas se adecuan al contexto de la UCIN, mientras que el empobrecimiento en las interacciones alrededor de los 24 días de internación, estaría en relación al estrés y el gasto de energía psíquica que supone la internación, las presiones y exigencias del ambiente médico, el alejamiento familiar y las dificultades económicas y sociales profundizadas por la prolongada hospitalización (Hauser, Milán, González \& Oiberman, 2017).

Pose (2004) postula que la experiencia vivida por las madres en la UCIN, los sentimientos y las fantasías que experimentaron dejan marcas que pueden influir en el vínculo con ese hijo. Field (1977, citado por Kreisler \& Soulé, 1990), afirma que debido a la separación de la madre y su hijo por la internación en la UCIN, la relación se establece en esas condiciones complejas con una madre poco preparada y un niño difícil. En esos momentos, el ritmo, la armonía y la sincronicidad necesarias en la relación pueden verse afectados. En la evolución de la relación intervienen factores como el apoyo familiar, la necesidad de la madre de volver a trabajar, la experiencia de otras separaciones, la personalidad de la madre, la posibilidad de contar con apoyo o ayuda especializada.

Luego de algunas semanas o incluso varios meses, si la evolución ha sido positiva, estas díadas madre-bebé egresarán de la terapia neonatal. Sin embargo, deberán continuar en contacto con los equipos de salud 
mediante un seguimiento especializado del desarrollo y el crecimiento de estos bebés aproximadamente hasta los 6 años. Desde un punto de vista psicológico, el alta implica una separación. Aquellas madres en quienes la prematurez y/o la patología del bebé provocaron sentimientos de desvalorización, de desconfianza sobre su capacidad maternal, de culpabilidad y frustración, luego del alta se encuentran en el hogar sumamente atemorizadas y sintiéndose incapaces de desempeñar su función materna. El vínculo con este bebé queda marcado por la fragilidad y la vulnerabilidad con la que será percibido por estos padres en el futuro (Pose, 2004). Estos sentimientos pueden obstaculizar el despliegue de fantasías y el investimento libidinal de ese bebé. En ese caso, los padres pueden adoptar una forma operatoria de cuidar al niño, privilegiando la atención de las necesidades físicas, pero sin poder investir emocionalmente su cuidado (Gutton, 1987).

Conocer lo que ocurre en los primeros momentos de la relación madre-hijo es crucial. La observación profesional atenta permitiría detectar con prontitud situaciones críticas, posibilitando el despliegue de intervenciones oportunas. Brazelton y Cramer (1993) remarcan la importancia de evaluar las interacciones madre-bebé durante la primera infancia, señalando que cuanto menor es el bebé las evaluaciones deben ser más frecuentes, como ocurre en el caso de los bebés prematuros y de riesgo. Los autores subrayan que factores subjetivos presentes en la madre pueden interrelacionarse de un modo particular con las características del bebé, creando interacciones distorsionadas que moldean la conducta del niño.

En el estudio que se informa, se pretendió conocer el modo en que se desplegaron las interacciones madre-bebé durante la internación en UCIN y posteriormente en los controles médicos en el Consultorio de Seguimiento para Niños de Alto Riesgo. Se expone el modo en que la técnica estadística de Análisis de Clúster, un procedimiento novedoso en nuestro medio, puede ser utilizado para agrupar a las díadas madre-bebé en función de las características de las interacciones.

\section{Método}

\section{Participantes}

La muestra estuvo conformada por 36 díadas mamá-bebé de alto riesgo. Se incluyeron díadas con bebés de ambos sexos que no superaran el mes de vida y que fueran considerados médicamente de alto riesgo por lo que debían permanecer internados. Se seleccionaron madres adolescentes o adultas, con o sin pareja al momento del nacimiento de su hijo. Respecto de los criterios de exclusión, no se incorporaron aquellos infantes que nacieron con un buen estado de salud, pero que debieron retornar al hospital luego del alta con alguna afección; tampoco se incorporaron díadas cuyas madres presentaban un diagnóstico previo de trastorno mental.

\section{Instrumentos}

Se utilizaron dos instrumentos para observar las interacciones madre-bebé durante la internación en la UCIN y los controles mensuales en el Consultorio de Seguimiento para Niños de Alto Riesgo.

La Escala de observación de vínculo madre-bebé internado en UCIN (N-EOV-INC) (Santos, 2010) indaga 6 funciones: acercamiento, contacto con la incubadora, corporal, visual, verbal, postural. Los ítems de cada una de las funciones tienen un puntaje asignado, dependiendo de que la conducta sea realizada por un solo miembro de la díada o por ambos. Al ser sumados los puntajes de cada ítem se obtiene el puntaje total de la función. Luego se adiciona el puntaje de todas las funciones obteniendo el puntaje total de la escala. Este valor se corresponde con un percentil y un puntaje $\mathrm{Z}$. El instrumento establece que un percentil de 25 o menos (puntajes entre 2 y 8) supone que la díada se encuentra en riesgo vincular, un 
percentil entre 30 y 40 (puntajes de 9) supone que la situación vincular es poco adecuada y un percentil superior o igual a 50 (puntajes entre 10 y 16) supone una situación vincular adecuada.

La Escala de Observación del Vínculo Madre-hijo (Oiberman, 2008) se aplica durante la consulta pediátrica y se divide en dos perfiles: de 0 a 6 meses (grilla 1) y de 7 a 15 meses (grilla 2). En el Perfil 1 se indaga la función corporal, visual y verbal; mientras que en el Perfil 2 a estas tres funciones se agrega la de sostén. Se consideran tanto las conductas maternas como las conductas del bebé y aquellas conductas de interacción mutua. En ambos perfiles los comportamientos de la madre y el bebé pueden tener un valor de 0 (ausencia de relación, ni el bebé ni la madre se comunican), 1 (solo uno de los miembros realiza la conducta sin obtener respuesta del otro) y 2 (ambos miembros realizan la conducta). Todos los puntajes obtenidos en la función se suman dando lugar a un puntaje total por función. En el Perfil 1, la interpretación de los valores varía en cada una de las funciones. Respecto de la función visual, los valores indican ausencia de comunicación (0 a 1 punto), comunicación inadecuada ( 2 a 3 puntos), comunicación buena (4 a 5 puntos) o muy buena (6 a 8 puntos). Respecto de la función corporal, los valores indican ausencia de comunicación ( 0 a 1 punto), comunicación inadecuada (2 puntos), comunicación buena ( 3 a 4 puntos) o muy buena (5 a 6 puntos). En cuanto a la función verbal, los valores indican ausencia de comunicación (0 punto), comunicación inadecuada (1 punto), comunicación buena (2 puntos) o muy buena (3 puntos). En el Perfil 2, en todas las funciones los puntajes suponen ausencia de comunicación (0 a 1 punto), comunicación buena (2 puntos) y comunicación muy buena (3 a 4 puntos). La escala no ofrece un puntaje general que incluya todas las funciones evaluadas.

\section{Procedimiento}

El presente trabajo forma parte de una investigación aprobada por el comité de ética de la Facultad de Psicología de la Universidad Nacional de San Luis (Argentina). A las madres se les explicó el objetivo y el procedimiento del estudio, aceptando ser parte del proyecto después de firmar un consentimiento informado por escrito.

Se realizaron observaciones vinculares mediante la aplicación de la escala de observación vincular N-EOV-INC durante el período de internación del bebé en la UCIN. Se realizaron las observaciones cuando la madre ingresaba en la UCIN para ver a su hijo mientras estaba en la incubadora. La primera observación de la díada debía realizarse durante el primer mes de vida del bebé, realizando las siguientes con intervalos de dos o tres días a fin de conocer si se producían variaciones con el transcurso del tiempo de internación, la maduración y el crecimiento del bebé. El tiempo de observación fue de 5 minutos, comenzando cuando la madre se ubicaba junto a la incubadora y finalizando al cumplirse los $5 \mathrm{mi}-$ nutos, o en el momento que la madre abandonaba la habitación.

En el seguimiento, se evaluaron las interacciones espontáneas madre-hijo que sucedieron en el contexto observacional natural del Consultorio de Seguimiento para Niños de Alto Riesgo. Se observó mensualmente a cada díada durante los 15 a 25 minutos de la visita pediátrica, utilizando la Escala de $\mathrm{Ob}$ servación del Vínculo Madre-hijo. Las interacciones fueron evaluadas entre octubre de 2011 y diciembre de 2014. La participación de las díadas en la investigación terminó cuando el niño tenía 12 meses de edad gestacional corregida. Los protocolos de observación fueron completados por un observador entrenado, inmediatamente después de cada observación.

\section{Análisis}

Se diseñó un sistema de codificación para distinguir los comportamientos mater- 
nos de los comportamientos infantiles. Se incluyeron los comportamientos de iniciación materna e infantil (corporal, visual, verbal y de sostén) como las respuestas del otro miembro de la díada. Se definieron 26 variables interactivas. En la UCIN estas variables fueron: Inicia la conducta Corporal el Bebé (ICB), Respuesta de la Madre ante el Contacto Corporal del bebé (rCM), Inicia la conducta Corporal la Madre (ICM), Respuesta del Bebé al Contacto Corporal materno (rCB), Inicia la conducta Visual el Bebé (IViB), Respuesta de la Madre a la Conducta Visual del bebé (rViM), Inicia la conducta Visual la Madre (IViM), Respuesta del bebé a la Conducta Visual materna (rViB), Inicia la conducta Verbal la Madre (IVeM), Respuesta del Bebé a la Conducta Verbal materna (rVeB). En el Consultorio de Seguimiento, a las variables mencionadas se agregaron: Inicia la conducta Verbal el Bebé (IVeBs), Respuesta de la Madre a la Conducta Verbal del bebé (rVeMs), Inicia la conducta de Sostén el Bebé (ISBs), Respuesta de la Madre a la Conducta de Sostén del bebé (rSMs), Inicia la conducta de Sostén la Madre (ISMs), Respuesta del Bebé a la Conducta de Sostén materno (rSBs).

En todas las variables, se otorgó un puntaje de 0 ante la ausencia de conducta interactiva o frente al rechazo de la conducta iniciada por el otro miembro de la díada. En cambio, se asignó 1 punto cada vez que se iniciaba una conducta interactiva o se respondía positivamente a la conducta propuesta por el otro miembro de la díada. Así por ejemplo, si en la primera observación la madre tocó al bebé dos veces, el puntaje en la variable ICM fue de 2, mientras que si el bebé permitió ser tocado solo una vez el puntaje en la variable rCB sería de 1. Esta organización de los datos permitió diferenciar con claridad a las díadas a partir de la frecuencia de las conductas interactivas.

Se calculó el valor medio por díada para cada variable y se realizó un Análisis de Clúster en el Software R. Este análisis consiste en la separación de los casos en grupos en función de las variables observadas en los datos. Se trata de una técnica estadística descriptiva que permite identificar grupos homogéneos de objetos o casos a los que se denomina clústeres, cuya traducción al español es la de grupos o racimos. Los casos que se ubican en un grupo específico comparten muchas características, por lo que tienen una alta semejanza, mientras que son muy disímiles de los casos que no pertenecen a ese grupo. El objetivo principal del análisis de clúster es discriminar, a partir de un conjunto previamente determinado de variables, grupos naturales de casos similares, por ejemplo grupos de díadas madrebebé. Cada clúster o grupo de casos puede ser caracterizado a partir del centro, que se entiende como el valor medio de todas las variables observadas. En esta investigación se utilizó la técnica $k$-means, la más recomendada para muestras de tamaño mediano, donde a partir de un número preestablecido de grupos se distribuyen los casos (Mooi \& Sarstedt, 2011).

A partir de este análisis se obtuvo el número de díadas que pertenecen a cada grupo así como los valores medios de cada grupo en cada una de las variables incluidas en el análisis. La pertenencia de las díadas a un grupo específico depende de la frecuencia con la que se presentaron las variables analizadas. El valor medio de cada variable representa el promedio de las conductas interactivas de esas díadas durante el total de las observaciones, por lo que se entiende que, a medida que aumenta el valor medio aumenta la cantidad de conductas interactivas propuestas por las díadas. Esto se corresponde con el porcentaje de respuesta del otro miembro de la díada, lo que da cuenta del nivel de encuentro de las díadas a lo largo de la interacción. Es decir, que la calidad de la interacción en cada grupo está en relación no solo a la cantidad de conductas propuestas por los miembros de las díadas, sino también al porcentaje de respuesta que hubo por parte del otro integrante. 


\section{Resultados}

En la Tabla 1 se presenta una descripción de los aspectos sociodemográficos de la muestra.

\section{Interacciones madre-bebé en UCIN}

Durante la internación en UCIN se realizó un total de 167 observaciones de las 36 díadas, a partir de las cuales se obtuvieron 3 grupos (clústeres) con las características que se detallan en la Tabla 2. En la Figura 1 se visualizan los grupos de díadas en función de las variables analizadas.

El grupo 1 "Interacciones Escasas", está integrado por las madres y los bebés que menos respondieron a las interacciones iniciadas por el otro miembro de la díada, además de ser estas madres las que propusieron la menor cantidad de conductas en comparación con el resto de la muestra. Esto se revierte cuando son los infantes los que inician las conductas, ya que presentaron las frecuencias más altas dentro de la muestra total. Se podría pensar que la escasa respuesta de los bebés podría estar relacionada con las pocas propuestas maternas. Estos infantes, frente a un ambiente poco alentador, buscarían de forma más reiterada interactuar con sus madres. Se puede señalar que en este grupo predominó el desencuentro, ya que las interacciones fueron escasas o no obtuvieron respuestas por parte del otro miembro de la díada, lo que indicaría que se trata del grupo en el que se observan mayores dificultades en las interacciones durante la internación en UCIN. Al realizar el análisis de las desviaciones estándares de las variables estudiadas, se observó que eran bajas, excepto para ICM y rCB (ICB: .530, rCM: .505, ICM: .933, rCB: .789, IViB: .323, rViM: .236, IViM: .514, rViB: .284, IVeM: .000, rVeB: .000).

En el grupo 2 "Interacciones Frecuentes", las madres iniciaron muchas conductas y los bebés respondieron en gran medida, especialmente a las propuestas corporales y verbales. Se trata del grupo de bebés que más respondieron a las iniciativas maternas. En cambio, estos infantes fueron los que menos propuestas realizaron dentro de la muestra total. Sin embargo, las madres respondieron siempre a las propuestas. Se podría pensar que la búsqueda intensa de la interacción por parte de las madres podría disminuir, en cierta medida, la iniciativa de los bebés. Se puede señalar que en este grupo existieron intentos de interactuar por parte de ambos miembros de la díada. Además, en general, se observa el porcentaje más elevado de respuestas maternas y de parte de los infantes dentro de la muestra. El análisis de la desviación estándar de las variables estudiadas arrojó los siguientes valores: ICB: .331, rCM: .331, ICM: .841, rCB: .854, IViB: .186, rViM: .186, IViM: .512, rViB: .627, IVeM: .658, rVeB: .675. Se observa que la desviación es relativamente baja, es decir, que los valores son bastante cercanos a la media para cada variable. Sin embargo, se registra un incremento de la desviación respecto de la media en las variables ICM, rCB, IViM, rViB, IVeM y rVeB, lo que indicaría que existe mayor diferencia en cuanto a la cantidad de conductas realizadas por las díadas.

En el grupo 3 "Interacciones Moderadas", las madres iniciaron intercambios y los bebés respondieron con frecuencia, especialmente a las verbalizaciones, aunque en menor medida en comparación con el grupo anterior, a los contactos corporales y las miradas. Al momento de iniciar las interacciones, los infantes recurrieron bastante a conductas corporales y visuales. Las madres respondieron, pero no a la totalidad de las conductas. Se puede señalar que en este grupo si bien las interacciones fueron bastante numerosas, existieron ciertos momentos de desencuentro, lo que se tradujo en la ausencia de respuesta del otro miembro de la díada. En este grupo, la cantidad de propuestas interactivas así como las respuestas, se ubican en un nivel intermedio en comparación con los dos grupos anteriores. El análisis de la desviación estándar de las variables estudiadas arrojó también valores relativamente bajos: ICB: .560, rCM: .556, 
ICM: .849, rCB: .785, IViB: .228, rViM: .199, IViM: .199, rViB: .199, IVeM: .615, rVeB: .615. Se percibe un incremento de la desviación en las variables ICB, rCM, ICM, $\mathrm{rCB}$, IVeM y rVeB, lo que indicaría mayor disparidad en la cantidad de conductas realizadas por las díadas.

\section{Interacciones madre-bebé en el Consultorio de Seguimiento para Niños de Alto Riesgo}

Se analizaron 223 observaciones realizadas a las 36 díadas durante el seguimiento en el Consultorio para Niños de Alto Riesgo, a partir de lo cual se agruparon las díadas en función de las características de las interacciones (ver Tabla 2). Las diferencias entre los tres grupos se visualizan en la Figura 2.

En el grupo 1 "Interacciones Desencontradas", se ubican las díadas que menos propiciaron los intercambios, lo que se observa en la exigua cantidad de conductas iniciadas. Estas madres respondieron en muy pocas oportunidades a las conductas de los infantes. En cambio, los bebés lo hicieron con mucha frecuencia, especialmente a nivel corporal, verbal y de sostén. A partir de esto se puede mencionar que las díadas de este grupo tuvieron dificultades al momento de iniciar la interacción, mientras que al responder a la conducta del otro miembro se observan diferencias: las madres no pudieron percibir los pocos intentos interactivos de los infantes y por lo tanto no respondieron en consonancia; en cambio, los niños captaron las conductas y respondieron considerablemente. El análisis de la desviación estándar de las variables estudiadas arrojó los siguientes valores: ICBs: .670, rCMs: .634, ICMs: .747, rCBs: .747, IViBs: 1.009, rViMs: .746, IViMs: .981, rViBs: .884, IVeBs: 1.191, rVeMs: .797, IVeMs: 1.254, rVeBs: .658, ISBs: .586, rSMs: .576, ISMs: .149 y rSBs: .149. Se observa que la desviación es relativamente alta respecto de la media para cada variable, en particular en IViBs, IVeBs y IVeMs, lo que indicaría mayor disparidad en la cantidad de conductas realizadas por los miembros de las díadas.

En el grupo 2 "Interacciones Sintonizadas", los intercambios fueron muy frecuentes. Las madres propusieron numerosas conductas a fin de interactuar con sus hijos, así como también respondieron en gran medida a los intentos de interacción de los infantes. Por su parte, los niños vocalizaron y/o emitieron sonidos de manera muy frecuente para captar la atención materna, buscaron tocar el cuerpo materno y ser sostenidos. Al momento de responder, este grupo de niños lo hizo con bastante frecuencia. Se puede decir entonces, que en este grupo existieron numerosos encuentros entre los miembros de las díadas, propiciados generalmente por las madres, ya que buscaron interactuar y captaron los intentos interactivos de los niños, quienes parecían más dispuestos a responder a la conducta materna y no tanto a iniciar por ellos mismos la interacción. El análisis de la desviación estándar de las variables estudiadas arrojó los siguientes valores: ICBs: .841, rCMs: .763, ICMs: 1.254 , rCBs: 1.254, IViBs: .830, rViMs: .678, IViMs: .992, rViBs: .927, IVeBs: 1.764, rVeMs: 1.125, IVeMs: 1.617, rVeBs: .848 , ISBs: .567 , rSMs: .567, ISMs: .359 y rSBs: .327. Se observa que la desviación es relativamente alta respecto de la media para cada variable, en particular en ICMs, rCBs, IVeBs, rVeMs y IVeMs, lo que indicaría mayor disparidad en la cantidad de conductas realizadas por los miembros de las díadas.

En el tercer grupo "Interacciones Vacilantes", los intercambios diádicos fueron bastante frecuentes y fluctuantes. Las madres propusieron con bastante periodicidad conductas a nivel corporal, visual y verbal. Por su parte, los infantes fueron quienes presentaron mayor cantidad de conductas interactivas en comparación con los niños de los otros grupos. Al momento de responder, tanto las madres como los niños mostraron ciertas diferencias en función del canal de comunicación escogido por el otro miembro de la díada, lo que se traduce en la variación en los percentiles de respuesta en cada caso. En este grupo se ubican los niños 
que mayor cantidad de conductas iniciaron, por lo que se podría inferir que estaría en relación con las fluctuaciones en la conducta materna. Se podría considerar que ciertos momentos de desajuste generarían el espacio para que los niños desplieguen su iniciativa en la interacción. El análisis de la desviación estándar de las variables estudiadas arrojó los siguientes valores: ICBs: .774, rCMs: .610, ICMs: 1.019, rCBs: 1.019, IViBs: .990, rViMs: .946, IViMs: .952, rViBs: .860, IVeBs: 1.221, rVeMs: .807, IVeMs: 1.378, rVeBs: .758, ISBs: 1.174, rSMs: .922, ISMs: .000 y rSBs: .000. Se observa que la desviación es relativamente alta respecto de la media para cada variable, en particular en ICMs, rCBs, IVeBs, IVeMs y ISBs, lo que indicaría mayor disparidad en la cantidad de conductas realizadas por los miembros de las díadas.

\section{Correlación entre las interacciones en UCIN y en el Consultorio de Seguimiento}

El análisis de Pearson arrojó que las respuestas visuales de los bebés en UCIN correlacionaron positivamente con las miradas $(r=1.00 ; p=.001)$ y las verbalizaciones $(r=.999 ; p=.030)$ propuestas por las madres durante las observaciones en el Consultorio de Seguimiento. En este sentido, la respuesta de los bebés estimularía la conducta materna. A su vez, las respuestas visuales de los infantes durante la internación correlacionaron positivamente con las vocalizaciones realizadas por los mismos durante el seguimiento ( $r=.999 ; p=.034)$.

En cambio, la iniciación de conductas visuales del bebé durante la internación correlacionó negativamente con sus respuestas visuales durante el seguimiento ( $r=-.998$; $p=.041$ ). Además, la iniciación de conductas visuales del bebé en UCIN correlacionó negativamente con las conductas visuales $(r=-.999 ; p=.025)$ y verbales $(r=-1.00$; $p=.005)$ propuestas por las madres. Asimismo, la iniciación visual en la UCIN por parte de los infantes correlacionó negativamente con sus verbalizaciones en el seguimiento $(r=-1.00 ; p=.009)$.

\section{Discusión}

Los resultados a los que se arribó en la presente investigación ponen en evidencia el carácter bidireccional y recíproco de todas las interacciones que se establecen desde el comienzo entre la madre y su hijo. El agrupamiento de las díadas, en función de las características de las interacciones a lo largo de 12 meses, pone en evidencia que algunas díadas tuvieron dificultades para interactuar tanto durante la estadía en la UCIN como durante los controles médicos en el Consultorio de Seguimiento. Por el contrario, otras díadas enfrentaron de manera más exitosa el contexto de la internación y lograron mantener interacciones gratificantes en la UCIN y en el Consultorio de Seguimiento para Niños de Alto Riesgo. Finalmente, otras díadas interactuaron de manera alternante durante el primer año de vida, ya sea porque establecieron interacciones satisfactorias durante la internación del bebé en la UCIN, aunque luego esas interacciones se empobrecieron notablemente durante el primer año de vida, o bien presentaron dificultades para interactuar en la terapia neonatal, las que disminuyeron considerablemente luego del alta, observándose interacciones frecuentes y gratificantes durante el seguimiento del infante en el primer año de vida.

Al considerar cada grupo en particular, se puede resaltar que el grupo 1 de la UCIN es el que tiene menor cantidad de interacciones, integrado por las madres que menor cantidad de conductas propusieron y los bebés que menos respondieron en comparación con el resto de la muestra. Esta situación se revierte llamativamente en cuanto a la búsqueda de conductas por parte de los bebés, ya que éstos son los que más propuestas realizaron en la muestra total; en cambio las madres son las que menos respondieron a la conducta de sus hijos. Se puede señalar entonces, que no solo las interacciones son escasas en este grupo, sino que también se observa un desencuentro entre las madres y los bebés. Las díadas 
que integraron este grupo en la UCIN continuaron con dificultades para interactuar en el Consultorio de Seguimiento. Esta imposibilidad de revertir las características de la interacción representaría un mayor riesgo para el desarrollo del vínculo y del bebé, tal como lo demuestra Arnold et al. (2013). Por su parte, Stern (1997) señala que las interacciones riesgosas se caracterizan por presentar más rupturas, discontinuidades y suelen ser menos variables. Esto se puso en evidencia en estas díadas, cuando sus miembros solían utilizar diferentes canales de comunicación a la hora de interactuar, por ejemplo en los controles en el Consultorio los niños buscaban predominantemente establecer contacto mediante sonidos, balbuceos y palabras con sus madres, mientras que ellas recurrían a las miradas como el modo privilegiado de interacción.

Resulta interesante subrayar que los infantes, que pertenecían a díadas que mantuvieron las dificultades para interactuar en la UCIN y en el Consultorio de Seguimiento, fueron aquellos que buscaron de manera más intensa establecer interacciones con sus madres durante la estadía en la UCIN. Sin embargo, al momento de las observaciones en el Consultorio de Seguimiento, estos niños redujeron notablemente los intentos de interacción, siendo parte del grupo que menos iniciativas propuso. En cambio, las madres mantuvieron su estilo de interacción caracterizado por escasos intentos de comunicarse con sus hijos y pocas respuestas frente a las propuestas de ellos, tanto en la internación como durante los controles médicos del primer año de vida. Este cambio, que se observó en las conductas propuestas por los infantes, podría estar en relación con lo sostenido por Nikodem (2009), quien afirma que los bebés poseen desde el nacimiento capacidades que les permiten realizar acciones en su entorno, que serán mantenidas a lo largo del tiempo y complejizadas en función de las respuesta que obtenga de los adultos significativos.

Los resultados de la presente investigación concuerdan con aquellos a los que arribaron Brazelton y Cramer (1993) sobre la llamada situación del rostro inexpresivo, donde el niño intenta en un primer momento suscitar respuestas en la madre, pero ante su ausencia, finalmente desvía la mirada y se retrae. De esta manera, la iniciativa de los infantes parecería haberse agotado, porque como sostiene Lebovici (citado por Huberman, 2003), el papel activo del bebé en la interacción, solo puede mantenerse por la respuesta recíproca de la madre, el rostro inexpresivo de ella tiene un efecto desorganizador en el niño.

Por otro lado, las díadas que pertenecieron a los grupos que establecieron intercambios conductuales y emocionales satisfactorios durante el primer año de vida del infante, habrían logrado sobreponerse a lo imprevisto de la situación de internación en UCIN y establecieron una vinculación satisfactoria para ambos miembros. Se trataría de díadas que han aprendido a relacionarse en una situación diferente a la esperada, en un contexto particular donde las interacciones estarán mediatizadas por elementos técnicos como respiradores, sondas, monitores. Durante las observaciones en el Consultorio de Seguimiento, estas díadas utilizaron diferentes canales de comunicación para interactuar, lo que permite afirmar, tomando los términos de Stern (1997), que se trataría de interacciones saludables porque son más sutiles y varían de un tema a otro con fluidez. Retomando también los aportes de Fava Vizziello, Zorzi y Bottos (1993), se puede considerar que se trataría de interacciones satisfactorias, donde las madres habrían podido interpretar las señales de los bebés y darles un significado, favoreciendo la autonomía y creatividad de sus hijos. Por su parte, los niños podrían explorar el mundo y las interacciones con placer, mostrando numerosos intereses e investimentos.

Además, durante los controles en el Consultorio de Seguimiento, se pudo observar que algunas madres de este grupo actuaron con mayor insistencia para contactarse con sus hijos, obtener respuestas por parte de ellos e incitarlos a participar en la interacción. Por momentos, la actitud materna se 
posicionó en un lugar central, conduciendo en mayor medida la comunicación y opacando las posibilidades del niño de desplegar su iniciativa. La conducta de iniciativa de estos infantes constituye, tal vez, un hallazgo en relación con los resultados obtenidos por otros investigadores en bebés de alto riesgo y prematuros. De esto surge el interrogante acerca de los efectos a largo plazo que podría tener en las iniciativas de los infantes, la conducta materna hiperactiva y con características de intrusividad, aun cuando los niños pueden iniciar por momentos las conductas interactivas.

Es interesante mencionar que en este grupo de díadas, las interacciones satisfactorias entre la madre y el infante no resultaron ser sincronizadas y recíprocas durante todo el tiempo de observación. Es decir, que no se observaron respuestas adecuadas de parte de la madre o el bebé a la totalidad de las conductas propuestas por el otro miembro de la díada. Por el contrario, siempre que se presentaron momentos de desajuste, ya sea a nivel corporal, verbal, visual o del sostén, en la mayoría de las oportunidades fueron corregidos garantizando la continuidad de la interacción. Esto coincide con lo expuesto por Duhalde et al. (2008), quienes afirman que lo más frecuente es que los encuentros sincrónicos ocurran sólo en pequeñas porciones del tiempo de interacción. Los desencuentros son frecuentes en la interacción diádica frente a los cuales se puede presentar la corrección como parte de un proceso mutuamente regulado.

Finalmente, se encontraron díadas que evidenciaron variaciones en las interacciones a lo largo de la investigación. Considerando los aportes de Fava Vizziello, De Rocco y Zingarello (1993), se puede concluir que las interacciones que son muy inestables durante los primeros meses de vida, están más relacionadas a situaciones reactivas que a la condición orgánica del niño y la estructura de personalidad de los padres. Para aquellas díadas que mantuvieron intercambios conductuales frecuentes y satisfactorios durante la estadía del infante en la
UCIN, pero luego fueron empobreciendo su estilo de interacción en el Consultorio de Seguimiento, se podría hipotetizar que los cambios estarían influidos por dos circunstancias. La primera estaría en relación a la aparición de dificultades en el desarrollo de estos infantes. Esto podría ser interpretado a partir de lo señalado por Stern (1997), quien sostiene que cuando se trata de un bebé con un retraso importante en el desarrollo, esta situación impide a los padres representarse a su hijo en el futuro, se ven invadidos por la incertidumbre, lo que podría incidir sobre la interacción. La otra circunstancia que podría haber incidido parecería estar más ligada al contexto familiar y social en que se desplegó la vinculación luego del egreso de la UCIN, dado que en el ambiente del hogar podrían existir conflictos familiares, de pareja, ausencia de apoyo de la madre por parte de la familia en la crianza del bebé.

Por el contrario, hubo díadas que mantuvieron interacciones poco frecuentes durante la internación en la UCIN, seguidas de un incremento de las interacciones luego del alta. En este grupo, se puede señalar que existieron impedimentos para lograr un encuentro conductual y emocional durante la internación del bebé, al decir de Lebovici (1983/1985) habrían existido dificultades en estas madres para adaptarse al bebé real. Las complicaciones que se observaron en los primeros momentos de encuentro madre-bebé internado parecerían estar referidas en particular al contexto de la internación. Posteriormente, la progresión positiva de las interacciones que se evidencia luego del alta en los controles en el Consultorio de Seguimiento, permite suponer que las díadas habrían requerido de más tiempo, así como del ambiente contenedor del hogar y de la familia, para establecer interacciones mutuamente provechosas y afectuosas. Esta afirmación coincide con lo planteado por Pose (2004) acerca de la importancia del proceso de duelo por el que deben atravesar los padres frente al nacimiento de un hijo prematuro y/o con patologías neonatales. 


\section{Conclusiones}

Los resultados de la presente investigación permiten concluir acerca de la importancia de estudiar las interacciones mamábebé. La detección temprana de las dificultades en la interacción permitiría implementar acciones adecuadas para favorecer el desarrollo del vínculo y del niño.

El vínculo temprano supone un proceso progresivo de construcción en el que participan la madre y su hijo, donde intervienen diversos factores que pueden facilitar o dificultar su sano desenvolvimiento. El nacimiento prematuro y/o con patología, así como la posterior internación del bebé desde los primeros momentos de vida en una Unidad de Cuidados Intensivos Neonatales, constituyen unas de las condiciones que han sido descriptas como posibles perturbadores de la vinculación madre-hijo. Sin embargo, a pesar de la conmoción inicial que provoca el nacimiento con dificultades, así como la visión del bebé en incubadora, en esta muestra la mayoría de las díadas estudiadas pudieron establecer interacciones satisfactorias a lo largo del primer año de vida.

La principal limitación de esta investigación es el tamaño y la heterogeneidad de la muestra, por lo que no es adecuado reali- zar generalizaciones de los resultados a otras díadas y en otros contextos. Las interacciones madre-bebé fueron observadas directamente sin la utilización de equipos de filmación, lo que dificulta la recodificación de los datos y la replicación del estudio. En investigaciones futuras sería interesante conocer si los tipos de interacciones propuestos se presentan con características similares en grupos diferentes y en díadas que se encuentran alojadas en Servicios de Neonatología que no responden al Modelo de Maternidades Centradas en la Familia.

La importancia de la aplicación del Análisis de Clúster en el campo de la investigación en Psicología, reside en la posibilidad de visualizar y agrupar de manera estadística, en nuestro caso, a las interacciones en función de sus características, en dos momentos diferentes como son la internación en UCIN y en el Consultorio de Seguimiento para Niños de Alto Riesgo. La utilización de esta técnica estadística descriptiva resulta novedosa en nuestro medio y en especial en el área de la Psicología Perinatal. Esta técnica permitiría organizar los datos de manera más eficiente y de esa manera, realizar un seguimiento de las interacciones madre-bebé a lo largo del tiempo. 
Interacciones madre-bebé. Análisis de clúster

\section{Tabla 1}

Aspectos sociodemográficos de la muestra

\begin{tabular}{|c|c|c|}
\hline \multicolumn{3}{|c|}{$n$} \\
\hline $\begin{array}{l}\text { Nivel educativo } \\
\text { materno }\end{array}$ & $\begin{array}{l}\text { Sin educación (2) } \\
\text { Estudios primarios (17) } \\
\text { Estudios secundarios (15) } \\
\text { Estudios terciarios/universitarios (2) }\end{array}$ & \\
\hline $\begin{array}{l}\text { Situación laboral } \\
\text { materna }\end{array}$ & $\begin{array}{l}\text { Ama de casa }(21) \\
\text { Desempleada (5) } \\
\text { Trabajo informal (7) } \\
\text { Trabajo formal (3) }\end{array}$ & \\
\hline $\begin{array}{l}\text { Motivo de internación } \\
\text { del bebé }\end{array}$ & $\begin{array}{l}\text { Enfermedad respiratoria ( } 21) \\
\text { Retardo en el crecimiento intrauterino (10) } \\
\text { Otros (5) }\end{array}$ & \\
\hline & $M$ & $D E$ \\
\hline $\begin{array}{l}\text { Edad Gestacional del } \\
\text { bebé al nacer (semanas) }\end{array}$ & $\begin{array}{l}32 \\
(\min 27, \max 41)\end{array}$ & 3.547 \\
\hline $\begin{array}{l}\text { Peso de nacimiento } \\
\text { (gramos) }\end{array}$ & 1.652 & .619521 \\
\hline $\begin{array}{l}\text { Tiempo de } \\
\text { hospitalización en } \\
\text { UCIN (días) }\end{array}$ & $\begin{array}{l}39 \\
(\min 9, \max 79)\end{array}$ & 19.724 \\
\hline Edad materna (años) & $\begin{array}{l}23 \\
(\min 16, \max 35)\end{array}$ & 5.111 \\
\hline
\end{tabular}


Tabla 2

Grupos de díadas a partir de las interacciones en UCIN y el Consultorio de Seguimiento

\begin{tabular}{|c|c|c|c|}
\hline \multicolumn{2}{|c|}{ UCIN } & \multicolumn{2}{|c|}{ Consultorio de Seguimiento } \\
\hline \multicolumn{2}{|c|}{ Grupo 1} & \multicolumn{2}{|c|}{ Grupo 1} \\
\hline Madre & Bebé & Madre & Bebé \\
\hline Corporal: $\mathrm{m}=.76$ & $89 \%$ de respuesta & Corporal: $\mathrm{m}=.57$ & $100 \%$ de respuesta \\
\hline Visual: $\mathrm{m}=.94$ & $6 \%$ de respuesta & Visual: $\mathrm{m}=1.13$ & $71 \%$ de respuesta \\
\hline \multirow[t]{2}{*}{ Verbal: $\mathrm{m}=0$} & \multirow[t]{2}{*}{-} & Verbal: $m=1.05$ & $31 \%$ de respuesta \\
\hline & & Sostén: $\mathrm{m}=.02$ & $100 \%$ de respuesta \\
\hline $77 \%$ de respuesta & Corporal: $\mathrm{m}=.34$ & $90 \%$ de respuesta & Corporal: $\mathrm{m}=.52$ \\
\hline \multirow[t]{3}{*}{$47 \%$ de respuesta } & Visual: $\mathrm{m}=.08$ & $66 \%$ de respuesta & Visual: $\mathrm{m}=1.01$ \\
\hline & & $50 \%$ de respuesta & Verbal: $\mathrm{m}=1.21$ \\
\hline & & $64 \%$ de respuesta & Sostén: $m=.56$ \\
\hline \multicolumn{2}{|c|}{ Grupo 2} & \multicolumn{2}{|c|}{ Grupo 2} \\
\hline Madre & Bebé & Madre & Bebé \\
\hline Corporal: $\mathrm{m}=1.13$ & $98 \%$ de respuesta & Corporal: $\mathrm{m}=1.41$ & $100 \%$ de respuesta \\
\hline Visual: $\mathrm{m}=1.31$ & $53 \%$ de respuesta & Visual: $\mathrm{m}=1.94$ & $83 \%$ de respuesta \\
\hline \multirow[t]{2}{*}{ Verbal: $\mathrm{m}=.89$} & \multirow[t]{2}{*}{$75 \%$ de respuesta } & Verbal: $\mathrm{m}=2.28$ & $28 \%$ de respuesta \\
\hline & & Sostén: $\mathrm{m}=.13$ & $85 \%$ de respuesta \\
\hline $100 \%$ de respuesta & Corporal: $\mathrm{m}=.10$ & $95 \%$ de respuesta & Corporal: $\mathrm{m}=.58$ \\
\hline \multirow[t]{3}{*}{$100 \%$ de respuesta } & Visual: $\mathrm{m}=.03$ & $72 \%$ de respuesta & Visual: $\mathrm{m}=.97$ \\
\hline & & $55 \%$ de respuesta & Verbal: $\mathrm{m}=1.55$ \\
\hline & & $100 \%$ de respuesta & Sostén: $\mathrm{m}=.72$ \\
\hline \multicolumn{2}{|c|}{ Grupo 3} & \multicolumn{2}{|c|}{ Grupo 3} \\
\hline Madre & Bebé & Madre & Bebé \\
\hline Corporal: $\mathrm{m}=2.10$ & $95 \%$ de respuesta & Corporal: $\mathrm{m}=1.20$ & $100 \%$ de respuesta \\
\hline Visual: $\mathrm{m}=1.25$ & $37 \%$ de respuesta & Visual: $m=1.65$ & $75 \%$ de respuesta \\
\hline \multirow[t]{2}{*}{ Verbal: $\mathrm{m}=.76$} & $85 \%$ de respuesta & Verbal: $m=1.79$ & $26 \%$ de respuesta \\
\hline & & Sostén: m= 0 & - \\
\hline $98 \%$ de respuesta & Corporal: $\mathrm{m}=.33$ & $95 \%$ de respuesta & Corporal: $\mathrm{m}=.67$ \\
\hline \multirow[t]{3}{*}{$85 \%$ de respuesta } & Visual: $\mathrm{m}=.05$ & $87 \%$ de respuesta & Visual: $\mathrm{m}=1.08$ \\
\hline & & $50 \%$ de respuesta & Verbal: $m=1.41$ \\
\hline & & $85 \%$ de respuesta & Sostén: $m=2.10$ \\
\hline
\end{tabular}




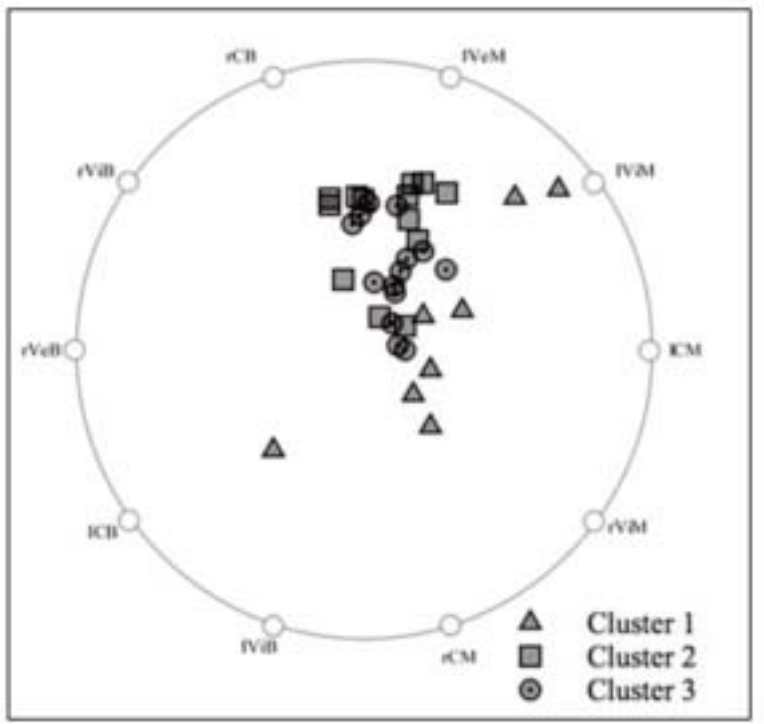

Figura 1. Grupos de díadas en función de las interacciones en UCIN. Cada símbolo indica una díada de la muestra. En la circunferencia externa de la figura están representadas de forma equidistante las variables analizadas en las interacciones.

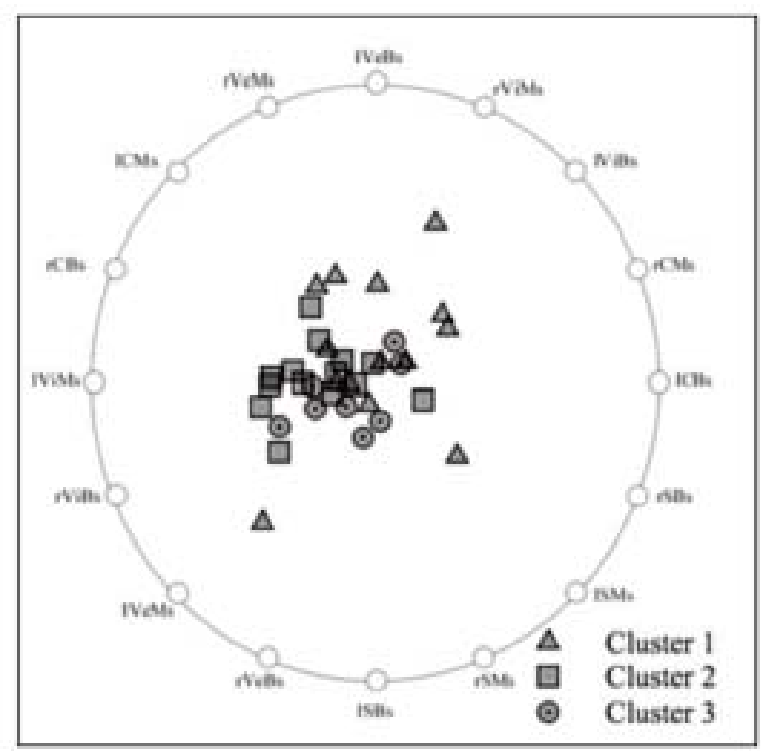

Figura 2. Grupos de díadas en función de las interacciones en el Consultorio de Seguimiento para Niños de Alto Riesgo.

Cada símbolo indica una díada de la muestra. En la circunferencia externa de la figura están representadas de forma equidistante las variables analizadas en las interacciones. 


\section{Referencias bibliográficas}

Arnold, L., Sawyer, A., Rabe, H., Abbott, J., Gyte, G., Duley, L. \& Ayers, S. (2013). Parents' first moments with their very preterm babies: a qualitative study. BMJ Open, 3, 1-6. http://dx.doi.org/10.1136/bmjopen2012-002487

Brazelton, T.B. \& Cramer, B.G. (1993). La relación más temprana. Padres, bebés y el drama del apego inicial. Barcelona: Paidós Ibérica.

Bruschweiler-Stern, N. (2009). Moments of meeting: Pivotal moments in mother, infant, father bonding: Switzerland. En J.K. Nugent, B.J. Petrauskas \& T.B. Brazelton (Eds.), The Newborn as a person: Enabling healthy infant development worldwide (pp. 70-84). United States: John Wiley \& Sons, Inc.

Davim, B.R.M., Enders, B.C. \& Silva, R.A.R. (2010). Mothers' feelings about breastfeeding their premature babies in a rooming-in facility. Revista da Escola de Enfermagem USP, 44(3), 713-718.

Duhalde, C., Huerin, V., Zucchi, A., Leonardelli, E., Mrahad, M.C., Silver, R.,... \& Schejtman, C. (2008). Regulación afectiva diádica y autorregulación afectiva del niño. Su relación con la autoestima y el funcionamiento reflexivo de la madre. En C. Schejtman (Comp.), Primera Infancia. Psicoanálisis e Investigación (pp.71-84). Buenos Aires, Argentina: Akadia Editorial.

Fava Vizziello, G. Zorzi, C. \& Bottos, M. (comp.) (1993). Los hijos de las máquinas: La vida de los niños internados en terapias intensivas neonatales (ed. español). Buenos Aires, Argentina: Nueva Visión.

Flacking, R., Lehtonen, L., Thomson, G. et al. (2012). Closeness and separation in neonatal intensive care. Acta Padiatrica, 101, 10321037.

Guía de Seguimiento del Recién Nacido de Riesgo (2da ed.) (2003). Dirección Nacional de Salud Materno Infantil. Buenos Aires: Ministerio de Salud, Presidencia de la Nación.

Guillaume, S., Michelin, N., Amrani, E. et al. (2013). Parents' expectations of staff in the early bonding process with their premature babies in the intensive care setting: a qualitative multicenter study with 60 parents. BMC Pediatrics, 13(18), 1-9.

Gutton, P. (1987). El bebé del psicoanalista. Perspectivas clínicas. Buenos Aires: Amorrortu.

Hauser, M.P., Milán, T.A., González, M.C. \& Oiberman, A. (2017). Incidencia del tiempo de internación en la terapia neonatal sobre las interacciones madre-bebé de alto riesgo. Estudos de Psicologia Campinas, 34(1), 131-140. http://dx.doi.org/10.1590/1982-02 752017000100013

Huberman, A. (2003). La madre deprimida y su bebé. Transmisión y génesis de la depresión en la primera infancia. Su tratamiento y prevención (pp. 167-177). En VI Jornadas del Departamento de Niñez y Adolescencia, APdeBA. Interpelaciones entre la clínica y la teoría. Buenos Aires, Argentina: APdeBA.

Kreisler, L. \& Soulé, M. (1990). El niño prematuro. En S. Lebovici, R. Diatkine \& M. Soulé, Tratado de Psiquiatría del Niño y del Adolescente (pp. 39-61). Tomo V. Madrid: Biblioteca Nueva.

Lebovici, S. (1985). El lactante, la madre y el psicoanalista: Las interacciones precoces. Buenos Aires, Argentina: Amorrortu (Trabajo Original publicado en 1983).

Leonardelli, E., Vernengo, M.P., Wainer, M. \& Duhalde, C. (2009). Desarrollo de un Modelo para la Observación Sistemática de Situaciones Interactivas lúdicas madreniño. En Memorias I Congreso Internacional de Investigación y Práctica Profesional en Psicología XVI Jornadas de Investigación Quinto Encuentro de Investigadores en Psicología del MERCOSUR (pp. 437-439). 
Tomo II. Buenos Aires: Ediciones de la Facultad de Psicología, Universidad de Buenos Aires.

Mooi, E. \& Sarstedt, M. (2011). A concise guide to market research: The process, data and methods using IBM SPSS statistics. Berlin: Springer. http://dx.doi.org/10.1007/ 978-3-642-12541-6_1

Nikodem, M.R. (2009). Niños de Alto Riesgo. Intervenciones tempranas en el Desarrollo y la Salud Infantil (1 ra ed.). Buenos Aires: Paidós.

Oiberman, A. (2008). Observando a los bebés: Técnicas vinculares madre-bebé, padrebebé (1 ra ed.). Buenos Aires, Argentina: Lugar Editorial.

Pose, G. (2004). Ser padres prematuros. El parto, el nacimiento y la relación con el bebé. Buenos Aires: Ediciones Del Candil.
Raznoszczyk de Schejtman, C. (2005). Regulación afectiva diádica madre-bebé y autorregulación del infante en la estructuración psíquica. Anuario de Investigaciones, XIII, 185-191.

Santos, M.S. (2010). Psychoneonatology: Results of a new observation scale of the bond mother-baby in incubator. In Advances in Perinatal Medicine. Proceedings (pp. 515518). Granada, España: Monduzzi Editore.

Stern, D. (1997). La constelación maternal: La psicoterapia en las relaciones entre padres e hijos (1ra ed.). Barcelona, España: Paidós Ibérica.
Recibido: 30 de marzo de 2017 Aceptado: 27 de septiembre de 2018 
